\title{
Nanostructured, Gd-doped Ceria Promoted by Pt or Pd: Investigation of the Electronic and Surface Structures and Relations to Chemical Properties
}

H. Borchert, Yu. Borchert, V. V. Kaichev, I. P. Prosvirin, G. M. Alikina, A. I. Lukashevich, V. I. Zaikovskii, E. M. Moroz, E. A. Paukshtis, V. I. Bukhtiyarov, and V. A. Sadykov

\section{Supporting Information}

\section{Fitting details for the analysis of XPS Ce 3d spectra}

XPS Ce $3 \mathrm{~d}$ spectra were deconvolved into five spin-orbit split doublets of Voigt functions, labeled $\mathrm{v}_{0} / \mathrm{u}_{0}, \ldots, \mathrm{v}^{\prime \prime \prime} / \mathrm{u}^{\prime \prime}$ in accordance to common use in the literature. For fitting, a combined polynomial and Shirley-type background function was used. The Gaussian width was constrained to be identical for the two spin-orbit split peaks of a given doublet, but allowed to vary from one doublet to another. The Lorentzian width was constrained to be identical for all ten components.

In a first step, the spectrum of the undoped cerium oxide sample was analyzed. A value of $0.75 \mathrm{eV}$ was found for the Lorentzian width. The spin-orbit splitting was constrained to be identical for the doublets $\mathrm{v}_{0} / \mathrm{u}_{0}, \ldots, \mathrm{v} / / \mathrm{u}$ " and found to be $18.57 \mathrm{eV}$. For the doublet $\mathrm{v} " / \mathrm{u} " ' \mathrm{a}$ slightly reduced value had to be used. The branching ratio was fixed to the theoretical value of $2 / 3$ for the doublets $v_{0} / u_{0}$, v/u and v"'/u'". However, for the doublets $v^{\prime} / u^{\prime}$ and $v^{\prime \prime} / u^{\prime \prime}$ a reduced value was necessary to obtain a good fit.

For the spectra of the Gd-doped ceria samples, the values of $0.75 \mathrm{eV}$ for the Lorentzian width and of $18.57 \mathrm{eV}$ for the spin orbit splitting of the doublets $\mathrm{v}_{0} / \mathrm{u}_{0}, \ldots, \mathrm{v} / \mathrm{u}$ " have been adapted from the analysis of the undoped sample. The spin-orbit splitting of the doublet v"'/u"' was found to be $18.41 \mathrm{eV} \pm 0.07 \mathrm{eV}$. The reduced value of the branching ratio for the doublets $\mathrm{v}^{\prime} / \mathrm{u}$ ' and $\mathrm{v}^{\prime \prime} / \mathrm{u}$ " was $0.57 \pm 0.05$.

Table S1 - Binding energies in $\mathrm{eV}$ for all components of the $\mathrm{Ce} 3 \mathrm{~d}$ photoemission spectra. The values in the lines marked as ' $\mathrm{O}_{2}$ ' and ' $\mathrm{CH}_{4}$ ' refer to results obtained after treatment of the samples with oxygen and methane, respectively.

\begin{tabular}{|c|c|c|c|c|c|c|c|c|c|c|c|}
\hline \multicolumn{2}{|l|}{ sample } & $\mathrm{v}_{0}$ & V & $\mathrm{V}^{\prime}$ & V" & v"' & $\mathrm{u}_{0}$ & u & $u^{\prime}$ & u" & $u^{\prime \prime \prime}$ \\
\hline \multirow[t]{2}{*}{$\mathrm{CeO}_{2-\mathrm{x}}$} & $\mathrm{O}_{2}$ & 880.1 & 882.5 & 884.9 & 888.6 & 898.3 & 898.7 & 901.1 & 903.4 & 907.2 & 916.7 \\
\hline & & & & & & & & & & & \\
\hline \multirow{2}{*}{$\mathrm{Pt} / \mathrm{Gd}_{0.2} \mathrm{Ce}_{0.8} \mathrm{O}_{2-\mathrm{x}}$} & $\mathrm{O}_{2}$ & -- & 882.5 & 884.2 & 888.7 & 898.4 & -- & 901.1 & 902.8 & 907.3 & 916.7 \\
\hline & $\mathrm{CH}_{4}$ & 880.3 & & & 888.6 & 898.3 & 898.8 & & 903.6 & 907.2 & 916.7 \\
\hline \multirow{2}{*}{$\mathrm{Pd} / \mathrm{Gd}_{0.2} \mathrm{Ce}_{0.8} \mathrm{O}_{2-\mathrm{x}}$} & $\mathrm{O}_{2}$ & 880.6 & 882.7 & 885.1 & 888.8 & 898.3 & 899.1 & 901.3 & 903.6 & 907.4 & 916.7 \\
\hline & $\mathrm{CH}_{4}$ & 880.5 & 882.6 & 884.9 & 888.6 & 898.4 & 899.0 & 901.2 & 903.5 & 907.2 & 916.7 \\
\hline \multirow{2}{*}{$\mathrm{Gd}_{0.4} \mathrm{Ce}_{0.6} \mathrm{O}_{2-\mathrm{x}}$} & $\mathrm{O}_{2}$ & -- & 882.8 & 884.9 & 888.7 & 898.3 & -- & 901 & 903.5 & 907.3 & 916.7 \\
\hline & $\mathrm{CH}_{4}$ & 880.1 & 882.7 & 884.7 & 888.5 & 898.2 & 898.7 & 901.2 & 903.2 & 907.1 & 916.7 \\
\hline \multirow{2}{*}{$\mathrm{Pt} / \mathrm{Gd}_{0.4} \mathrm{Ce}_{0.6} \mathrm{O}_{2-\mathrm{x}}$} & $\mathrm{O}_{2}$ & -- & 882.6 & 884.1 & 888.5 & 898.3 & -- & 901.2 & 902.6 & 907.1 & 916.7 \\
\hline & $\mathrm{CH}_{4}$ & 880.3 & 882.5 & 884.8 & 888.4 & 898.3 & 898.8 & 901.1 & 903.4 & 907.0 & 916.7 \\
\hline \multirow{2}{*}{$\mathrm{Pd} / \mathrm{Gd}_{0.4} \mathrm{Ce}_{0.6} \mathrm{O}_{2-\mathrm{x}}$} & $\mathrm{O}_{2}$ & 880.5 & 882.5 & 884.8 & 888.6 & 898.3 & 899 & 901 & 903.4 & 907.1 & 916.7 \\
\hline & $\mathrm{CH}_{4}$ & 880.5 & 882.5 & 884.8 & 888.6 & 898.3 & 899.0 & 901.1 & 903.4 & 907.1 & 916.7 \\
\hline
\end{tabular}


Table S2 - Results for the Gaussian width, the spin-orbit splitting (SO), and the branching ratio (BR).

\begin{tabular}{|c|c|c|c|c|c|c|c|c|c|c|}
\hline \multirow{2}{*}{\multicolumn{2}{|c|}{ sample }} & \multicolumn{5}{|c|}{ Gaussian width [eV] } & \multicolumn{2}{|c|}{$\mathrm{SO}[\mathrm{eV}]$} & \multicolumn{2}{|c|}{ BR } \\
\hline & & $\mathrm{v}_{0} / \mathrm{u}_{0}$ & $\mathrm{v} / \mathrm{u}$ & $v^{\prime} / u^{\prime}$ & v"/u" & v'"/u'" & v'"/u'" & $\begin{array}{c}\text { other } \\
\text { doublets }\end{array}$ & $\begin{array}{l}\text { v'/u', } \\
v^{\prime \prime} / u^{\prime \prime}\end{array}$ & $\begin{array}{l}\text { other } \\
\text { doublets }\end{array}$ \\
\hline \multirow[b]{2}{*}{$\mathrm{CeO}_{2-\mathrm{x}}$} & & & & & & & & & & \\
\hline & $\mathrm{O}_{2}$ & 2.5 & 2.9 & 2.4 & 5.1 & 3.5 & 18.39 & 18.57 & 0.51 & 0.67 \\
\hline \multirow[b]{2}{*}{$\mathrm{Pt} / \mathrm{Gd}_{0.2} \mathrm{Ce}_{0.8} \mathrm{O}_{2-\mathrm{x}}$} & $\mathrm{O}_{2}$ & -- & 4.5 & 2.7 & 6.5 & 4.6 & 18.34 & 18.57 & 0.55 & 0.67 \\
\hline & $\mathrm{CH}_{4}$ & 1.7 & 2.6 & 2.5 & 4.1 & 2.8 & 18.40 & 18.57 & 0.53 & 0.67 \\
\hline \multirow{2}{*}{$\mathrm{Pd} / \mathrm{Gd}_{0.2} \mathrm{Ce}_{0.8} \mathrm{O}_{2-\mathrm{x}}$} & $\mathrm{O}_{2}$ & 1.6 & 2.5 & 2.9 & 4.2 & 3.0 & 18.41 & 18.57 & 0.50 & 0.67 \\
\hline & $\mathrm{CH}_{4}$ & 2.0 & 2.5 & 4.1 & 4.7 & 3.4 & 18.26 & 18.57 & 0.63 & 0.67 \\
\hline \multirow{2}{*}{$\mathrm{Gd}_{0.4} \mathrm{Ce}_{0.6} \mathrm{O}_{2-\mathrm{x}}$} & $\mathrm{O}_{2}$ & -- & 3.4 & 2.9 & 5.2 & 3.4 & 18.42 & 18.57 & 0.56 & 0.67 \\
\hline & $\mathrm{CH}_{4}$ & 1.3 & 3.1 & 3.2 & 5.3 & 3.3 & 18.48 & 18.57 & 0.54 & 0.67 \\
\hline \multirow{2}{*}{$\mathrm{Pt} / \mathrm{Gd}_{0.4} \mathrm{Ce}_{0.6} \mathrm{O}_{2-\mathrm{x}}$} & $\mathrm{O}_{2}$ & $\begin{array}{l}-- \\
\end{array}$ & 3.8 & 2.4 & 6.3 & 3.9 & 18.39 & 18.57 & 0.58 & 0.67 \\
\hline & $\mathrm{CH}_{4}$ & 1.4 & 2.7 & 3.3 & 5.1 & 2.9 & 18.39 & 18.57 & 0.62 & 0.67 \\
\hline \multirow{2}{*}{$\mathrm{Pd} / \mathrm{Gd}_{0.4} \mathrm{Ce}_{0.6} \mathrm{O}_{2-\mathrm{x}}$} & $\mathrm{O}_{2}$ & 3.4 & 4.0 & 6.5 & 7.4 & 4.7 & 18.37 & 18.57 & 0.67 & 0.67 \\
\hline & $\mathrm{CH}_{4}$ & 2.1 & 2.4 & 4.0 & 4.6 & 2.9 & 18.37 & 18.57 & 0.58 & 0.67 \\
\hline
\end{tabular}

\section{XPS Gd 4d spectra}

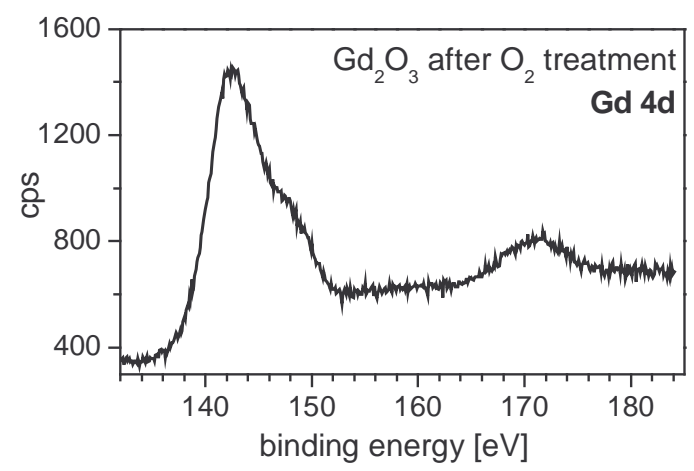

Figure S1 - XPS Gd $4 d$ spectrum of the pure gadolinium oxide sample after the oxidation pretreatment. 